\title{
PREVALENNCIA DE DOENÇAS CRÔNICAS EM IDOSOS ATENDIDOS NA ÁREA DE ABRANGÊNCIA DA ESTRATÉGIA SAÚDE DA FAMILIA NO INTERIOR DO RS
}

\author{
Jéssica Andressa Rizzardo ${ }^{1}$ \\ Juliane Bervian² \\ Gustavo Cavalcanti3 \\ Ana Luísa Sant'Anna Alves ${ }^{4}$ \\ resumo
}

Objetivo: avaliar a prevalência de doenças crônicas em idosos atendidos na Estratégia de Saúde da Família em um município do norte do estado do Rio Grande do Sul. Método: trata-se de um estudo transversal de dados secundários, realizado com indivíduos de 60 anos ou mais, através de prontuários eletrônicos do Sistema de

\footnotetext{
1 Graduada em Nutrição. Nutricionista. E-mail: nutrição.jar@gmail.com.

2 Graduada em Odontologia. Doutora em Odontologia. Professora Titular da Universidade de Passo Fundo (UPF), vinculada ao Departamento de Odontopediatria e Saúde Coletiva. E-mail: jbervian@ upf.br.

3 Graduado em Enfermagem. Mestre em Envelhecimento Humano. Professor Substituto Temporário da Universidade de Passo Fundo (UPF), vinculado ao Curso de Enfermagem. E-mail: gustavocavalcanti@ upf.br.

4 Graduada em Nutrição. Doutora em Epidemiologia. Professora Titular Il da Universidade de Passo Fundo (UPF), vinculada ao Programa de Pós-Graduação em Envelhecimento Humano. E-mail: alves. als@upf.br.
} 
Informação do Sistema Único de Saúde, no período de janeiro a dezembro de 2016. As doenças crônicas investigadas foram diabetes mellitus, hipertensão arterial sistêmica, acidente vascular cerebral, infarto, câncer e ainda a presença de multimorbidade para 2 ou mais doenças associadas. Também foram coletadas informações demográficas, internação nos últimos 12 meses e uso de tabaco. Os dados foram analisados através de software de estatística. Para as variáveis quantitativas foram calculadas as medidas de tendência central e dispersão, e para as variáveis qualitativas foram apresentas as frequências absolutas e relativas simples. Para as associações foi aplicado o Teste exato de Fisher e o Teste Qui-quadrado. Resultados: as doenças crônicas com maior prevalência foram hipertensão arterial sistêmica (37,3\%) e diabetes mellitus (10,1\%), simultaneamente hipertensão arterial sistêmica e diabetes mellitus 9,1\%, e a prevalência de multimorbidade foi de 11\%. A média de idade foi de 70,02 anos $(+7,93)$, sendo que a maioria tinha entre 60 a 69 anos $(55,2 \%)$, eram do sexo feminino (57,7\%), não haviam internado nos últimos 12 meses $(98,6 \%)$ e não faziam uso de tabaco $(97,3 \%)$. Foi observado maior prevalência de multimorbidade entre os idosos com 80 anos ou mais ( $p<0,001)$, e a associação entre doenças e sexo, a maior prevalência de Hipertensão Arterial foi no sexo feminino e maior prevalência de Infarto Agudo do Miocárdio no sexo masculino $(p<0,05)$. Conclusão: os dados do estudo mostraram uma baixa prevalência de doenças crônicas e multimorbidade.

palavras-chave

Idosos. Doenças Crônicas. Diabetes Mellitus. Hipertensão Arterial Sistêmica. Multimorbidade.

O envelhecimento populacional se mostra como um grande desafio para a sociedade, pois promoverá um incremento das demandas sociais e econômicas em todo o mundo. Em 2010, no Brasil (IBGE, 2016), havia aproximadamente 39 idosos para cada grupo de 100 jovens, e as estimativas para 2040 é que essa parcela da população represente em torno de 153 idosos para cada 100 jovens (MIRANDA; MENDES; SILVA, 2016). 
A transição epidemiológica juntamente com o envelhecimento populacional resulta na redução de mortes por doenças infecciosas e incrementa a carga de doenças crônicas - constituindo um problema de saúde de maior magnitude. Responsáveis por $72 \%$ das causas de morte, as doenças crônicas que se destacam são as relacionadas ao aparelho circulatório (31,3\%), câncer $(16,3 \%)$, diabetes $(5,2 \%)$ e doença respiratória crônica $(5,8 \%)$, com maior destaque em grupos vulneráveis, como idosos com baixa renda e escolaridade (BRASIL, 2011).

A ocorrência de doenças crônicas está associada a fatores de risco como tabagismo, álcool, inatividade física e alimentação não saudável (DUNCAN et al., 2012; BAUMGARTEL et al., 2016; BERNAL et al., 2017; SILVA et al., 2017), níveis elevados de colesterol, baixo consumo de frutas e verduras e sedentarismo, os quais são fatores responsáveis pela epidemia de sobrepeso e obesidade, pela elevada prevalência de hipertensão arterial e pelo colesterol alto (BRASIL, 2011; IBGE, 2014).

De acordo com a Pesquisa Nacional de Saúde (PNS) de 2013, do total de entrevistados, 45,1\% tinham pelo menos uma doença crônica. Desta população que apresentava pelo menos uma doença crônica, a maior prevalência era em mulheres (50,4\%), da região sul do país (52,1\%) (MALTA et al., 2015). Anterior a esta pesquisa nacional, a presença de várias doenças crônicas associadas foi mencionada por Campolina, Dini e Ciconelli (2011) que identificaram 14,7\% da população investigada com duas doenças crônicas e 5,1\%, tinham três ou mais doenças crônicas. A multimorbidade é citada em estudos como um enorme desafio para os serviços de saúde no mundo, indicando prevalências que variam de 50 a 98\% (MARENGONI et al., 2011; FORTIN et al., 2012).

A multimorbidade, isto é, a ocorrência de duas ou mais doenças crônicas em um indivíduo é frequente nos idosos (HARRISON et al., 2014) e resulta na piora da qualidade de vida, com maior risco de morte, o aumento na procura por serviços de saúde, maiores custos com internações prolongadas, frequentes e recorrentes, além de contribuir para a fragilidade e incapacidades funcionais nos idosos (BOYD; FORTIN, 2010; BATISTA, 2014). Nessa perspectiva, o objetivo do estudo foi verificar a prevalência e fatores associados a doenças crônicas em idosos na área de abrangência da Estratégia Saúde da Família do município de Marau/RS. 
Trata-se de um estudo transversal com utilização de dados secundários, de prontuários eletrônicos de idosos atendidos na Estratégia Saúde da Família de um município no interior do Rio Grande do Sul. O período de coleta de dados foi de 01 de janeiro a 31 de dezembro de 2016. Foram incluídos dados de prontuário eletrônico de 3.958 indivíduos com 60 anos ou mais com cadastro ativo no sistema informatizado de atenção básica do município. Este estudo foi aprovado pelo Comitê de Ética em Pesquisa da Universidade de Passo Fundo sob parecer no ${ }^{\circ}$.034.194.

As doenças crônicas investigadas foram diabetes mellitus (DM), hipertensão arterial sistêmica (HAS), acidente vascular cerebral (AVC), infarto agudo do miocárdio (IAM), câncer (CA) e ainda a presença de multimorbidade (ocorrência de 2 doenças ou mais). Ainda foram coletadas informações demográficas, internação nos últimos 12 meses e uso de tabaco.

Os dados foram analisados em software de estatística (SPSS 18), para as variáveis quantitativas foram calculadas as medidas de tendência central e dispersão e para as variáveis qualitativas foram apresentas as frequências absolutas e relativas simples com intervalo de confiança de $95 \%$. Para as associações foi aplicado o Teste exato de Fisher e o Teste Qui-quadrado.

\section{Resultados}

Foram investigados os prontuários de 3.958 idosos atendidos na Estratégia Saúde da Família. A maioria era do sexo feminino (57,7\%), com média de idade de 70,02 anos (+7,93), a maioria com idade entre 60 a 69 anos $(55,2 \%)$ não fumava $(97,3 \%)$ e não foi internada nos últimos 12 meses (98,6\%) (Tabela 1). 
Tabela 1 - Caracterização da amostra quanto às variáveis demográficas, uso de tabaco e internação de idosos atendidos na Estratégia Saúde da Família de Marau, RS, 2016 (n=3958).

\begin{tabular}{lll}
\hline Variáveis & Categorias & $\mathbf{n}(\%)$ \\
\hline Sexo & Masculino & $1674(42,3 \%)$ \\
& Feminino & $2284(57,7 \%)$ \\
Faixa etária & 60 a 69 anos & $2184(55,2 \%)$ \\
& 70 a 79 anos & $1226(31,0 \%)$ \\
& 80 anos ou mais & $548(13,8 \%)$ \\
Uso de tabaco & Não & $3851(97,3 \%)$ \\
Internação nos & Sim & $107(2,7 \%)$ \\
últimos 12 meses & Não & $3903(98,6 \%)$ \\
& Sim & $55(1,4 \%)$ \\
\hline
\end{tabular}

Fonte: Elaborada pelos autores.

As doenças crônicas com maior prevalência foram HAS (37,3\%) e DM $(10,1 \%)$, sendo que a prevalência simultânea de DM e HAS foi de 9,1\% ( $n=360,18)$. As demais doenças (acidente vascular cerebral, câncer e infarto agudo do miocárdio) apresentaram prevalência abaixo de $2 \%$. Quanto à multimorbidade, a prevalência foi de $11 \%$ (Tabela 2).

Tabela 2 - Descrição da presença de doenças e multimorbidade de idosos atendidos na Estratégia Saúde da Família de Marau, RS, 2016 (n=3958).

\begin{tabular}{lll}
\hline Variáveis & Categorias & $\mathbf{n}(\%)$ \\
\hline Hipertensão Arterial Sistêmica (HAS) & Não & $2482(62,7 \%)$ \\
Dim & $1476(37,3 \%)$ \\
Diabetes Mellitus (DM) & Não & $3558(89,9 \%)$ \\
Infarto Agudo do Miocárdio (IAM) & Sim & $400(10,1 \%)$ \\
& Não & $3930(99,3 \%)$ \\
Acidente Vascular Cerebral (AVC) & Sim & $28(0,7 \%)$ \\
& Não & $3904(98,6 \%)$ \\
& Sim & $54(1,4 \%)$
\end{tabular}




\begin{tabular}{lll}
\hline Variáveis & Categorias & n (\%) \\
\hline Câncer $(\mathrm{CA})$ & Não & $3885(98,2 \%)$ \\
& Sim & $73(1,8 \%)$ \\
Multimorbidade & Não & $3521(89,0 \%)$ \\
& Sim & $437(11,0 \%)$ \\
\hline
\end{tabular}

Fonte: Elaborada pelos autores.

No que diz respeito à associação entre doenças crônicas, multimorbidades e faixa etária, foi observado maior prevalência de multimorbidade entre os idosos com 80 anos ou mais ( $\mathrm{p}<0,001)$. A mesma tendência foi observada para HAS, DM e AVC $(<0,001)$ (Tabela 3$)$.

Tabela 3 - Associação entre doenças crônicas, multimorbidade e faixa etária de idosos atendidos na Estratégia Saúde da Família no município de Marau, RS, 2016 (n=3958).

Idade

\begin{tabular}{|c|c|c|c|c|c|}
\hline \multirow[t]{2}{*}{ Doenças } & & \multirow{2}{*}{$\begin{array}{c}60 \text { a } 69 \text { anos } \\
n(\%)\end{array}$} & \multirow{2}{*}{$\begin{array}{c}70 \text { a } 79 \text { anos } \\
\text { n (\%) }\end{array}$} & \multirow{2}{*}{$\begin{array}{c}80 \text { anos ou }+ \\
n(\%)\end{array}$} & \multirow[t]{2}{*}{$p$-valor } \\
\hline & & & & & \\
\hline \multirow[t]{2}{*}{ Multimorbidade } & Não & $2003(91,7 \%)$ & $1057(86,2 \%)$ & $461(84,1 \%)$ & $<0,001$ \\
\hline & Sim & 181 (8,3\%) & $169(13,8 \%)$ & $87(15,9 \%)$ & \\
\hline \multirow[t]{2}{*}{ HAS } & Não & $1510(69,1 \%)$ & $695(56,7 \%)$ & $277(27,1 \%)$ & $<0,001$ \\
\hline & Sim & $674(30,9 \%)$ & $531(43,3 \%)$ & 50,5 (49,5\%) & \\
\hline \multirow[t]{2}{*}{ DM } & Não & 2009 (92,0\%) & $1072(87,4 \%)$ & $477(87,0 \%)$ & $<0,001$ \\
\hline & Sim & 175 (8,0\%) & $154(12,6 \%)$ & $71(13,0 \%)$ & \\
\hline \multirow[t]{2}{*}{ AVC } & Não & 2167 (99,2\%) & 1205 (98,3\%) & $532(97,1 \%)$ & $<0,001$ \\
\hline & Sim & $17(0,8 \%)$ & $21(1,7 \%)$ & $16(2,9 \%)$ & \\
\hline \multirow[t]{2}{*}{ IAM } & Não & $2171(99,4 \%)$ & $1218(99,3 \%)$ & $541(98,7 \%)$ & 0,226 \\
\hline & Sim & $13(0,6 \%)$ & $8(0,7 \%)$ & $7(1,3 \%)$ & \\
\hline \multirow[t]{2}{*}{ CA } & Não & $2149(98,4 \%)$ & 1198 (97,7\%) & 538 (98,2\%) & 0,365 \\
\hline & Sim & $35(1,6 \%)$ & $28(2,3 \%)$ & $10(1,8 \%)$ & \\
\hline
\end{tabular}

Fonte: Elaborada pelos autores. 
Quanto à associação entre doenças e sexo foi observado maior prevalência de hipertensão arterial no sexo feminino e maior prevalência de Infarto Agudo do Miocárdio no sexo masculino $(\mathrm{p}<0,05)$ (Tabela 4).

Tabela 4 - Associação entre doenças e sexo de idosos atendidos na Estratégia Saúde da Família de Marau, RS, 2016 (n=3958).

\begin{tabular}{|c|c|c|c|c|}
\hline \multirow{3}{*}{ Doenças } & \multicolumn{3}{|c|}{ Sexo } & \multirow{3}{*}{$\mathrm{p}$-valor } \\
\hline & & Masculino & Feminino & \\
\hline & & $\mathrm{n}(\%)$ & n (\%) & \\
\hline \multirow[t]{2}{*}{ Multimorbidade } & Não & $1492(89,1 \%)$ & 2029 (88,8\%) & ${ }^{\star *} 0,406$ \\
\hline & Sim & $182(10,9 \%)$ & $255(11,2 \%)$ & \\
\hline \multirow[t]{2}{*}{ HAS } & Não & $1088(65,0 \%)$ & $1394(61,0 \%)$ & 0,006 \\
\hline & Sim & $586(35,0 \%)$ & $890(39,0 \%)$ & \\
\hline \multirow[t]{2}{*}{ DM } & Não & $1509(90,1 \%)$ & $2049(89,7 \%)$ & 0,348 \\
\hline & Sim & $165(9,9 \%)$ & $235(10,3 \%)$ & \\
\hline \multirow[t]{2}{*}{ AVC } & Não & $1649(98,5 \%)$ & $2255(98,7 \%)$ & 0,321 \\
\hline & Sim & $25(1,5 \%)$ & $29(1,3 \%)$ & \\
\hline \multirow[t]{2}{*}{ IAM } & Não & $1657(99,0 \%)$ & $2273(99,5 \%)$ & 0,038 \\
\hline & Sim & $17(1,0 \%)$ & $11(0,5 \%)$ & \\
\hline \multirow[t]{2}{*}{ CA } & Não & 1641 (98,0\%) & $2244(98,2 \%)$ & 0,347 \\
\hline & Sim & $33(2,0 \%)$ & $40(1,8 \%)$ & \\
\hline
\end{tabular}

Fonte: Elaborada pelos autores.

$p^{\star \star}$ Teste exato de Fischer

4 Discussão

O presente estudo identificou baixa prevalência de doenças crônicas quando comparado com estudos nacionais, sendo $37,3 \%$ de HAS e $10,1 \%$ de DM. No entanto, a prevalência simultânea de DM e HAS foi de 9,1\%. De acordo 
com a Organização Mundial da Saúde (WHO, 2011), essas doenças são fatores de risco importantes para complicações cardíacas e cerebrovasculares.

Outros resultados são evidenciados na Pesquisa Nacional de Acesso, Utilização e Promoção do Uso Racional de Medicamentos, de caráter transversal e com amostra probabilística populacional em municípios brasileiros urbanos (AKERMAN; FREITAS, 2017), bem como em outros estudos (GRAMANI, 2014; RAMOS et al., 2016) em que a prevalência encontrada de hipertensão arterial sistêmica foi de 59\% com aumento significativo no avançar da idade, 66\% no grupo com mais de 80 anos de idade (RAMOS et al., 2016). Por tratar-se de estudo populacional, representativos de regiões brasileiras encontramos diferença considerável quando comparado aos resultados do presente estudo. Tais diferenças podem ser justificadas pelo estudo de Gramani (2014) que avaliou dificuldades em todas as regiões quanto ao atendimento de atenção básica, contudo, nas regiões norte e nordeste há baixo número de profissionais e de graduados nas áreas da Saúde, já nas regiões sul e sudeste, as carências envolvem falta de investimento financeiro. Porém, de forma comparativa, estão melhores, assim, as dificuldades podem refletir nos problemas de saúde da população atendida.

Quanto à presença de DM, em um estudo com idosos participantes dos Inquéritos de Saúde no Município de São Paulo (ISA-Capital) em 2003 e 2008, foi verificada prevalência de 17,6\% e 20,1\% (STOPA et al., 2014), respectivamente. Os resultados sobressaem os encontrados no presente estudo, podendo ser justificado pela diferença de acesso aos serviços públicos de saúde para controle da doença.

Ainda sobre a prevalência de DM e HAS, em um estudo transversal de base populacional e abrangência nacional, HAS e DM foram as DCNT de maior prevalência entre indivíduos com 60 anos ou mais, representando 50,6\% e 18,1\% respectivamente (THEME FILHA et al., 2015). No que tange a prevalência simultânea de HAS e DM, estudo nacional com idosos evidenciou que entre os que residem nas regiões Sul, Sudeste e Centro-Oeste apresentaram a maior prevalência simultânea de HAS e DM (17,0\%), no entanto mais elevada do que a prevalência observada no presente estudo (FRANCISCO et al., 2018). Esse fato pode ser justificado, uma vez que, no município de Marau a cobertura de Estratégia Saúde Família é de 100\% e está implementada e consolidada desde o ano de 2002. Ressalta-se que o local de pesquisa é uma cidade de pequeno porte com 40.629 habitantes, com Índice de Desenvolvimento Humano (IDHM) em 0,83, o valor do Produto Interno Bruto (PIB) em R\$1.107.505,89, e o PIB per capita em R $\$ 30.849,75$ (IBGE, 2016), proporcionando desta forma uma melhor qualidade de vida e atenção à saúde a todos os seus cidadãos. 
Diante disso, a multimorbidade comum em idosos, que pode ser a associação de DM e HAS ou de outras doenças, está relacionada a vários fatores, como de aumento da demanda por cuidados em saúde, capacidade funcional reduzida, maior mortalidade, além de maiores custos (BATISTA, 2014). No presente estudo, a prevalência de multimorbidade observada foi de $11 \%$, sendo entre os idosos com 80 anos ou mais de idade.

No entanto, em estudo sobre a prevalência de multimorbidade de acordo com características socioeconômicas e demográficas, observou que multimorbidade acomete $51,2 \%$ de pessoas acima de 60 anos, em sua maioria, mulheres moradoras da zona urbana (CARVALHO et al., 2017). Na amostra de idosos de Marau, a prevalência foi inferior, contudo a variável multimorbidade pode ter sido subestimada, uma vez que foram investigados um número reduzido de doenças para compor este desfecho, além disso, a realidade observada no país como um todo não reflete a cobertura de atendimento na Atenção Básica de municípios de pequeno porte.

Na Pesquisa Nacional de Saúde, foram avaliadas dez doenças crônicas não transmissíveis, encontrou-se prevalência de $47,1 \%$ de duas doenças em indivíduos com 60 anos ou mais e 33,2\% com três doenças ou mais (THEME FILHA et al., 2015). Assim, as prevalências de multimorbidade podem ser muito diferentes, pois dependem do número de doenças que são incluídas no estudo. Estudo similar foi realizado no município de Porto Alegre, em que avaliaram prontuários eletrônicos das ESF. Os autores evidenciaram que $47,5 \%$ do total de idosos, referiram duas ou mais doenças crônicas, sendo que o percentual aumentava conforme a idade (SILVA et al., 2017). Apesar das diferenças encontradas quanto ao número de doenças incluídas no estudo, o maior alcance de ações de controle, vigilância e monitoramento e atenção aos indivíduos portadores de doenças crônicas observadas no município de Marau podem ter refletido na prevalência de multimorbidade.

Diante de tais evidências, com o aumento da população idosa, um atendimento especializado para acolher o paciente em relação às doenças crônicas é fundamental para o adequado manejo da demanda na atenção básica. De acordo com a Pesquisa Nacional de Saúde (PNS) de 2013, o percentual de pessoas que procuraram atendimento de saúde com 60 anos ou mais de idade foi de 25\% (IBGE, 2016).

Projeção realizada pelo IBGE aponta que 18,6\% da população em 2030 terá 60 anos de idade ou mais, em 2060, de 33,7\% da população representará essa faixa etária. Em 2014, esse indicador foi mais elevado na Região Sul, onde $15,2 \%$ da população era representada por idosos (IBGE, 2016). Considerar o envelhecimento e longevidade populacional é determinante para o adequado 
planejamento do sistema de seguridade social, em especial no que compete à saúde (GOTTLIEB et al., 2011).

Apesar dos resultados encontrados, as interpretações devem ser realizadas com cautela, uma vez que, trata-se de dados secundários, muitas vezes preenchidos sem a devida acurácia de informações. Além disso, poucas variáveis encontravam-se disponíveis no prontuário eletrônico e reduzido número de doenças foram incluídas para avaliar multimorbidade. Tais problemas, no entanto, refletem o delineamento transversal, que interfere no registro da doença, o que pode subestimar prevalências, sugere-se um estudo longitudinal com informações importantes e que possam pautar o planejamento de políticas públicas a este grupo de usuários.

\section{Conclusão}

Foi observada baixa prevalência de doenças crônicas, e verificou-se que dentre as doenças crônicas, as de maior prevalência neste estudo foram a Hipertensão Arterial Sistêmica e a Diabetes Mellitus. Em relação a ocorrência de 2 doenças crônicas ou mais no mesmo usuário, evidenciou-se que a prevalência de multimorbidade aumentou com a idade, que pode ser um fator fisiológico pelo próprio processo de envelhecimento. Foi encontrada associação entre a maior prevalência de hipertensão arterial no sexo feminino e a maior prevalência de Infarto Agudo do Miocárdio no sexo masculino. Salienta-se, portanto, que estudos sobre doenças crônicas devem ser encorajados para contribuir com intervenções adequadas na prevenção de doenças, promoção e proteção da saúde do idoso, considerando a demanda crescente dessa faixa etária nos serviços de saúde. 
abstract

Objective: to evaluate the prevalence of chronic diseases in older adults served in the Family Health Strategy in the city in the north of the state of Rio Grande do Sul. Method: this is a cross-sectional study of secondary data, carried out with individuals aged 60 years or older, using electronic records of the Information System of the Unified Health System, in the period from January to December 2016. Chronic diseases investigated were diabetes mellitus, systemic arterial hypertension, stroke, heart attack, cancer and the presence of multimorbidity for 2 or more associated diseases. We also collected demographic information, hospitalization in the last 12 months and use of tobacco. The data were analyzed with statistical software, for the quantitative variables were calculated the measures of central tendency and dispersion; and for the qualitative variables were presented the absolute frequencies and simple relatives. For associations it was applied the Fisher exact test and Chi-square test. Results: the most prevalent chronic diseases were systemic arterial hypertension (37.3\%) and diabetes mellitus (10.1\%), simultaneously systemic arterial hypertension and diabetes mellitus (9.1\%), and the prevalence of multimorbidity was $11 \%$. The mean age was 70.02 years (+7.93), the majority were between 60 and 69 years old (55.2\%), were female (57.7\%), did not smoke $(97,3 \%)$ and had not hospitalized in the last 12 months (98.6\%). Results: the most prevalent chronic diseases were systemic arterial hypertension (37.3\%) and diabetes mellitus (10.1\%), both systemic arterial hypertension and diabetes mellitus 9.1\%, and the prevalence of multimorbidity was $11 \%$. A higher prevalence of multimorbidity was observed among older adults with 80 years or more ( $p<0.001)$, and the association between diseases and sex, the highest prevalence of Arterial Hypertension was in females and a higher prevalence of acute myocardial infarction in males ( $p<0.05$ ). Conclusion: the study data showed a low prevalence of chronic diseases and multimorbidity.

keywords

Older Adults. Chronic Diseases. Diabetes Mellitus. Systemic Arterial Hypertension. Multimorbidity. 
AKERMAN, Marco; FREITAS, Osvaldo de. Pesquisa Nacional sobre Acesso, Utilização e Promoção do Uso Racional de Medicamentos (PNAUM): avaliação dos serviços de atenção farmacêutica primária. Revista de Saúde Pública, São Paulo, v. 51, supl. 2, p. 1-5, 2017.

BATISTA, Sandro Rodrigues. A complexidade da multimorbidade. Journal of Management \& Primary Health Care, Brasil, v. 5, n. 1, p. 125-126, 2014.

BAUMGARTEL, Carine et al. Fatores de risco e proteção de doenças crônicas em adultos: estudo de base populacional em uma cidade de médio porte no sul do Brasil. Revista Brasileira de Medicina de Família e Comunidade, Rio de Janeiro, v. 11, n. 38, p. 1-13, 2016

BERNAL, Regina Tomie Ivata et al. Sistema de vigilância de fatores de risco e proteção para doenças crônicas por inquérito telefônico (Vigitel): mudança na metodologia de ponderação. Epidemiologia e Serviços de Saúde, Brasília, DF, v. 26, n. 4, p. 701-712, 2017.

BOYD, Cynthia M.; FORTIN, Martin. Future of multimorbidity research: how should understanding of multimorbidity inform health system design? Public Health Reviews, v. 32, n. 2, p. 451-474, 2010.

BRASIL. Ministério da Saúde. Secretaria de Vigilância em Saúde. Plano de ações estratégicas para o enfrentamento das doenças crônicas não transmissíveis (DCNT) no Brasil: 2011-2022. Brasília, DF: Ministério da Saúde, 2011.

CAMPOLINA, Alessandro Gonçalves; DINI, Patrícia Skolaude; CICONELLI, Rozana Mesquita. Impacto da doença crônica na qualidade de vida de idosos da comunidade em São Paulo (SP, Brasil). Ciência \& Saúde Coletiva, Rio de Janeiro, v. 16, n. 6, p. 2919-2925, 2011.

CARVALHO, Januse Nogueira de et al. Prevalence of multimorbidity in the Brazilian adult population according to socioeconomic and demographic characteristics. Plos One, San Francisco, v. 12, n. 4, e0174322, 2017.

DUNCAN, Bruce Bartholow et al. Doenças crônicas não transmissíveis no Brasil: prioridade para enfrentamento e investigação. Revista de Saúde Pública, São Paulo, v. 46, supl. 1, p. 126-134, 2012.

FORTIN, Martin et al. A systematic review of prevalence studies on multimorbidity: toward a more uniform methodology. The Annals of Family Medicine, United States, v. 10, n. 2, p. 142-151, 2012.

FRANCISCO, Priscila Maria Stolses Bergamo et al. Prevalência simultânea de hipertensão e diabetes em idosos brasileiros: desigualdades individuais e contextuais. Ciência \& Saúde Coletiva, Rio de Janeiro, v. 23, n. 11, p. 3829-3840, 2018. Disponível em: http:// www.scielo.br/scielo.php?script=sci_abstract\&pid=S1413-81232018001103829\&lng= pt\&nrm=iso. Acesso em: 29 jun. 2019.

GOTTLIEB, Maria Gabriela Valle et al. Envelhecimento e longevidade no Rio Grande do Sul: um perfil histórico, étnico e de morbi-mortalidade dos idosos. Revista Brasileira de Geriatria e Gerontologia, Rio de Janeiro, v. 14, n. 2, p. 365-380, 2011.

GRAMANI, Maria Cristina. Inter-regional performance of the public health system in a high-inequality country. Plos One, San Francisco, v. 9, n. 1, e86687, 2014.

HARRISON, Christopher et al. Examining different measures of multimorbidity, using a large prospective cross-sectional study in Australian general practice. BMJ Open, London, v. 4, n. 7, e004694, 2014.

INSTITUTO BRASILEIRO DE GEOGRAFIA E ESTATÍSTICA (IBGE). Pesquisa nacional por amostra de domicilios contínua, 2014. Rio de Janeiro: IBGE, 2014 
INSTITUTO BRASILEIRO DE GEOGRAFIA E ESTATÍSTICA (IBGE). Síntese de indicadores sociais: uma análise das condições de vida da população brasileira - 2016. Rio de Janeiro: IBGE, 2016

MALTA, Deborah Carvalho et al. A vigilância e o monitoramento das principais doenças crônicas não transmissíveis no Brasil - Pesquisa Nacional de Saúde, 2013. Revista Brasileira de Epidemiologia, São Paulo, v. 18, supl. 2, p. 3-16, 2015.

MARENGONI, Alessandra et al. Aging with multimorbidity: a systematic review of the literature. Ageing Research Reviews, Netherlands, v. 10, n. 4, p. 430-439, 2011.

MIRANDA, Gabriella Morais Duarte; MENDES, Antonio da Cruz Gouveia; SILVA, Ana Lucia Andrade da. O envelhecimento populacional brasileiro: desafios e consequências sociais atuais e futuras. Revista Brasileira de Geriatria e Gerontologia, Rio de Janeiro, v. 19, n. 3, p. 507-519, 2016.

RAMOS, Luiz Roberto et al. Polifarmácia e polimorbidade em idosos no Brasil: um desafio em saúde pública. Revista de Saúde Pública, São Paulo, v. 50, supl. 2, p. 1-13, 2016.

SILVA, Amanda Ramalho et al. Doenças crônicas não transmissíveis e fatores sociodemográficos associados a sintomas de depressão em idosos. Jornal Brasileiro de Psiquiatria, Rio de Janeiro, v. 66, n. 1, p. 45-51, 2017.

STOPA, Sheila Rizzato et al. Diabetes autorreferido em idosos: comparação das prevalências e medidas de controle. Revista de Saúde Pública, São Paulo, v. 48, n. 4, p. 554-562, 2014

THEME FILHA, Mariza Miranda et al. Prevalência de doenças crônicas não transmissíveis e associação com autoavaliação de saúde: Pesquisa Nacional de Saúde, 2013. Revista Brasileira de Epidemiologia, São Paulo, v. 18, supl. 2, p. 83-96, 2015.

WORLD HEALTH ORGANIZATION (WHO). Global atlas on cardiovascular disease prevention and control. Edited by Shanthi Mendis, Pekka Puska and Bo Norrving. Geneva: WHO, 2011.

Data de Submissão: 13/06/2018

Data de Aprovação: 14/06/2019 
